May 30, 2003

\title{
Density-functional theory calculations of aqueous redox potentials of fourth-period transition metals
}

\author{
Merle Uudsemaa and Toomas Tamm* \\ Department of Chemistry, \\ Tallinn Technical University, \\ Ehitajate tee 5, EE-19086, Estonia.
}

\section{Supporting information}

*Correspondence to: T. Tamm; e-mail: toomas.tamm@ttu.ee 
Table S1: Components of calculated redox potentials using different models.

\begin{tabular}{|c|c|c|c|c|c|c|c|}
\hline System & Model & $\mathrm{IP}(\mathrm{g})$ & $E_{\text {sol }}$ & $T \Delta S$ & $\Delta E_{S H E}$ & $\begin{array}{l}E_{\text {redox }} \\
\text { calc. }\end{array}$ & $\begin{array}{l}E_{\text {redox }} \\
\text { expt. }\end{array}$ \\
\hline \multirow[t]{6}{*}{$\mathrm{Sc}^{3+} / \mathrm{Sc}^{2+}$} & ion & 25.72 & 0 & 0.48 & -4.43 & 21.77 & -2.3 \\
\hline & ion + contin. & 25.72 & -15.89 & 0.48 & -4.43 & 5.88 & -2.3 \\
\hline & ion $+6 \mathrm{H}_{2} \mathrm{O}$ & 13.19 & 0 & 0.48 & -4.43 & 9.24 & -2.3 \\
\hline & ion $+6 \mathrm{H}_{2} \mathrm{O}+$ contin. & 13.19 & -9.99 & 0.48 & -4.43 & -0.75 & -2.3 \\
\hline & ion $+18 \mathrm{H}_{2} \mathrm{O}$ & 9.11 & 0 & 0.48 & -4.43 & 5.16 & -2.3 \\
\hline & ion $+18 \mathrm{H}_{2} \mathrm{O}+$ contin. & 9.11 & -6.77 & 0.48 & -4.43 & -1.61 & -2.3 \\
\hline \multirow[t]{6}{*}{$\mathrm{Ti}^{3+} / \mathrm{Ti}^{2+}$} & ion & 28.65 & 0 & 0.45 & -4.43 & 24.67 & -0.9 \\
\hline & ion + contin. & 28.65 & -15.87 & 0.45 & -4.43 & 8.80 & -0.9 \\
\hline & ion $+6 \mathrm{H}_{2} \mathrm{O}$ & 14.18 & 0 & 0.45 & -4.43 & 10.20 & -0.9 \\
\hline & ion $+6 \mathrm{H}_{2} \mathrm{O}+$ contin. & 14.18 & -10.11 & 0.45 & -4.43 & 0.09 & -0.9 \\
\hline & ion $+18 \mathrm{H}_{2} \mathrm{O}$ & 10.05 & 0 & 0.45 & -4.43 & 6.07 & -0.9 \\
\hline & ion $+18 \mathrm{H}_{2} \mathrm{O}+$ contin. & 10.05 & -6.82 & 0.45 & -4.43 & -0.75 & -0.9 \\
\hline \multirow[t]{6}{*}{$\mathrm{V}^{3+} / \mathrm{V}^{2+}$} & ion & 30.00 & 0 & 0.45 & -4.43 & 26.02 & -0.255 \\
\hline & ion + contin. & 30.00 & -15.70 & 0.45 & -4.43 & 10.33 & -0.255 \\
\hline & ion $+6 \mathrm{H}_{2} \mathrm{O}$ & 15.21 & 0 & 0.45 & -4.43 & 11.23 & -0.255 \\
\hline & ion $+6 \mathrm{H}_{2} \mathrm{O}+$ contin. & 15.21 & -10.23 & 0.45 & -4.43 & 1.00 & -0.255 \\
\hline & ion $+18 \mathrm{H}_{2} \mathrm{O}$ & 10.61 & 0 & 0.45 & -4.43 & 6.63 & -0.255 \\
\hline & ion $+18 \mathrm{H}_{2} \mathrm{O}+$ contin. & 10.61 & -6.80 & 0.45 & -4.43 & -0.17 & -0.255 \\
\hline
\end{tabular}


Table S1 (continued)

\begin{tabular}{|c|c|c|c|c|c|c|c|}
\hline System & Model & $\mathrm{IP}(\mathrm{g})$ & $E_{\text {sol }}$ & $T \Delta S$ & $\Delta E_{S H E}$ & $\begin{array}{l}E_{\text {redox }} \\
\text { calc. }\end{array}$ & $\begin{array}{l}E_{\text {redox }} \\
\text { expt. }\end{array}$ \\
\hline \multirow[t]{6}{*}{$\mathrm{Cr}^{3+} / \mathrm{Cr}^{2+}$} & ion & 31.10 & 0 & 0.42 & -4.43 & 27.09 & -0.42 \\
\hline & ion + contin & 31.10 & -15.90 & 0.42 & -4.43 & 11.19 & -0.42 \\
\hline & ion $+6 \mathrm{H}_{2} \mathrm{O}$ & 15.10 & 0 & 0.42 & -4.43 & 11.09 & -0.42 \\
\hline & ion $+6 \mathrm{H}_{2} \mathrm{O}+$ contin. & 15.10 & -10.37 & 0.42 & -4.43 & 0.72 & -0.42 \\
\hline & ion $+18 \mathrm{H}_{2} \mathrm{O}$ & 10.42 & 0 & 0.42 & -4.43 & 6.41 & -0.42 \\
\hline & ion $+18 \mathrm{H}_{2} \mathrm{O}+$ contin. & 10.42 & -6.91 & 0.42 & -4.43 & -0.50 & -0.42 \\
\hline \multirow[t]{6}{*}{$\mathrm{Mn}^{3+} / \mathrm{Mn}^{2+}$} & ion & 33.95 & 0 & 0.54 & -4.43 & 30.06 & 1.54 \\
\hline & ion + contin. & 33.95 & -15.91 & 0.54 & -4.43 & 14.15 & 1.54 \\
\hline & ion $+6 \mathrm{H}_{2} \mathrm{O}$ & 16.95 & 0 & 0.54 & -4.43 & 13.06 & 1.54 \\
\hline & ion $+6 \mathrm{H}_{2} \mathrm{O}+$ contin. & 16.95 & -10.35 & 0.54 & -4.43 & 2.70 & 1.54 \\
\hline & ion $+18 \mathrm{H}_{2} \mathrm{O}$ & 11.96 & 0 & 0.54 & -4.43 & 8.07 & 1.54 \\
\hline & ion $+18 \mathrm{H}_{2} \mathrm{O}+$ contin. & 11.96 & -6.85 & 0.54 & -4.43 & 1.21 & 1.54 \\
\hline \multirow[t]{6}{*}{$\mathrm{Fe}^{3+} / \mathrm{Fe}^{2+}$} & ion & 31.97 & 0 & 0.35 & -4.43 & 27.89 & 0.77 \\
\hline & ion + contin. & 31.97 & -15.91 & 0.35 & -4.43 & 11.98 & 0.77 \\
\hline & ion $+6 \mathrm{H}_{2} \mathrm{O}$ & 16.16 & 0 & 0.35 & -4.43 & 12.08 & 0.77 \\
\hline & ion $+6 \mathrm{H}_{2} \mathrm{O}+$ contin. & 16.16 & -10.16 & 0.35 & -4.43 & 1.92 & 0.77 \\
\hline & ion $+18 \mathrm{H}_{2} \mathrm{O}$ & 11.63 & 0 & 0.35 & -4.43 & 7.55 & 0.77 \\
\hline & ion $+18 \mathrm{H}_{2} \mathrm{O}+$ contin. & 11.63 & -6.83 & 0.35 & -4.43 & 0.73 & 0.77 \\
\hline
\end{tabular}


Table S1 (continued)

\begin{tabular}{llllllll}
\hline System & Model & $\mathrm{IP}(\mathrm{g})$ & $E_{\text {sol }}$ & $T \Delta S$ & $\Delta E_{S H E}$ & $\begin{array}{c}E_{\text {redox }} \\
\text { calc. }\end{array}$ & $\begin{array}{l}E_{\text {redox }} \\
\text { expt. }\end{array}$ \\
\hline $\mathrm{Co}^{3+} / \mathrm{Co}^{2+}$ & & & & & & \\
& ion $\mathrm{Co}^{3+}$ Mult=1 & 38.25 & 0 & 0.37 & -4.43 & 34.19 & 1.92 \\
& ion + contin. & 38.25 & -15.91 & 0.37 & -4.43 & 18.27 & 1.92 \\
& ion $+6 \mathrm{H}_{2} \mathrm{O}$ & 17.34 & 0 & 0.37 & -4.43 & 13.28 & 1.92 \\
& ion $+6 \mathrm{H}_{2} \mathrm{O}+$ contin. & 17.34 & -10.78 & 0.37 & -4.43 & 2.50 & 1.92 \\
& ion $+18 \mathrm{H}_{2} \mathrm{O}$ & 12.15 & 0 & 0.37 & -4.43 & 8.09 & 1.92 \\
& ion $+18 \mathrm{H}_{2} \mathrm{O}+$ contin. & 12.15 & -6.99 & 0.37 & -4.43 & 1.10 & 1.92 \\
& & & & & & \\
& ion $\mathrm{Co}^{3+} \mathrm{Mult=5}$ & 34.68 & 0 & 0.37 & -4.43 & 30.62 & 1.92 \\
& ion $+\mathrm{contin}^{3}$ & 34.68 & -15.91 & 0.37 & -4.43 & 14.70 & 1.92 \\
& ion $+6 \mathrm{H}_{2} \mathrm{O}$ & 17.30 & 0 & 0.37 & -4.43 & 13.24 & 1.92 \\
& ion $+6 \mathrm{H}_{2} \mathrm{O}+$ contin. & 17.30 & -10.16 & 0.37 & -4.43 & 3.09 & 1.92 \\
& ion $+18 \mathrm{H}_{2} \mathrm{O}$ & 12.59 & 0 & 0.37 & -4.43 & 8.53 & 1.92 \\
& ion $+18 \mathrm{H}_{2} \mathrm{O}+$ contin. & 12.59 & -6.78 & 0.37 & -4.43 & 1.75 & 1.92 \\
\hline
\end{tabular}


Table S1 (continued)

\begin{tabular}{|c|c|c|c|c|c|c|c|}
\hline System & Model & $\operatorname{IP}(g)$ & $E_{\text {sol }}$ & $T \Delta S$ & $\Delta E_{S H E}$ & $\begin{array}{l}E_{\text {redox }} \\
\text { calc. }\end{array}$ & $\begin{array}{l}E_{\text {redox }} \\
\text { expt. }\end{array}$ \\
\hline \multirow[t]{12}{*}{$\mathrm{Ni}^{3+} / \mathrm{Ni}^{2+}$} & ion $\mathrm{Ni}^{3+}$ Mult $=2$ & 37.58 & 0 & 0.33 & -4.43 & 33.48 & 2.3 \\
\hline & ion + contin. & 37.58 & -16.07 & 0.33 & -4.43 & 17.40 & 2.3 \\
\hline & ion $+6 \mathrm{H}_{2} \mathrm{O}$ & 18.21 & 0 & 0.33 & -4.43 & 14.11 & 2.3 \\
\hline & ion $+6 \mathrm{H}_{2} \mathrm{O}+$ contin. & 18.21 & -10.53 & 0.33 & -4.43 & 3.58 & 2.3 \\
\hline & ion $+18 \mathrm{H}_{2} \mathrm{O}$ & 12.94 & 0 & 0.33 & -4.43 & 8.84 & 2.3 \\
\hline & ion $+18 \mathrm{H}_{2} \mathrm{O}+$ contin. & 12.94 & -6.90 & 0.33 & -4.43 & 1.94 & 2.3 \\
\hline & ion $\mathrm{Ni}^{3+}$ Mult $=4$ & 36.06 & 0 & 0.33 & -4.43 & 31.96 & 2.3 \\
\hline & ion + contin. & 36.06 & -15.92 & 0.33 & -4.43 & 16.04 & 2.3 \\
\hline & ion $+6 \mathrm{H}_{2} \mathrm{O}$ & 18.15 & 0 & 0.33 & -4.43 & 14.05 & 2.3 \\
\hline & ion $+6 \mathrm{H}_{2} \mathrm{O}+$ contin. & 18.15 & -10.08 & 0.33 & -4.43 & 3.97 & 2.3 \\
\hline & ion $+18 \mathrm{H}_{2} \mathrm{O}$ & 13.21 & 0 & 0.33 & -4.43 & 9.11 & 2.3 \\
\hline & ion $+18 \mathrm{H}_{2} \mathrm{O}+$ contin. & 13.21 & -6.87 & 0.33 & -4.43 & 2.24 & 2.3 \\
\hline \multirow[t]{6}{*}{$\mathrm{Cu}^{3+} / \mathrm{Cu}^{2+}$} & ion & 37.25 & 0 & 0.45 & -4.43 & 33.27 & 2.4 \\
\hline & ion + contin. & 37.25 & -15.91 & 0.45 & -4.43 & 17.36 & 2.4 \\
\hline & ion $+6 \mathrm{H}_{2} \mathrm{O}$ & 17.87 & 0 & 0.45 & -4.43 & 13.89 & 2.4 \\
\hline & ion $+6 \mathrm{H}_{2} \mathrm{O}+$ contin. & 17.87 & -10.24 & 0.45 & -4.43 & 3.66 & 2.4 \\
\hline & ion $+18 \mathrm{H}_{2} \mathrm{O}$ & 13.20 & 0 & 0.45 & -4.43 & 9.22 & 2.4 \\
\hline & ion $+18 \mathrm{H}_{2} \mathrm{O}+$ contin. & 13.20 & -6.91 & 0.45 & -4.43 & 2.32 & 2.4 \\
\hline
\end{tabular}


Table S2: Energies (in Hartrees) of the calculated complexes

\begin{tabular}{llll}
\hline File name & DFT energy & ZPE & DFT + COSMO \\
\hline Co2+4_18h2o-B & -2759.36073712962 & 0.4600947 & -2759.59860464513 \\
Co2+4_6h2o_Ci & -1841.37279961073 & 0.1506025 & -1841.67640649466 \\
Co3+1_18h2o_B & -2758.90643828562 & 0.4522624 & -2759.40109982296 \\
Co3+1_6h2o_Ci-S6 & -1840.7378651951 & 0.1527856 & -1841.43776157991 \\
Co3+5_18h2o_B & -2758.88786065228 & 0.4498065 & -2759.37484142234 \\
Co3+5_6h2o_Ci & -1840.7348580429 & 0.1486141 & -1841.41181059629 \\
Cr2+5_18h2o_B & -2421.00676825432 & 0.4597885 & -2421.24246386843 \\
Cr2+5_6h2o_Ci & -1503.02356706988 & 0.1527509 & -1503.32479569343 \\
Cr3+4_18h2o_B & -2420.6228571273 & 0.4586323 & -2421.11248792614 \\
Cr3+4_6h2o_Ci & -1502.46863864339 & 0.1526725 & -1503.15088439102 \\
Cu2+2_18h2o_B & -3017.08357220928 & 0.4533696 & -3017.3208927676 \\
Cu2+2_6h2o_Ci & -2099.09430037235 & 0.1526605 & -2099.40302157408 \\
Cu3+3_18h2o_B & -3016.59352524424 & 0.4485229 & -3017.08465534058 \\
Cu3+3_6h2o_S6 & -2098.43274297809 & 0.1479696 & -2099.11759175564 \\
Fe2+5_18h2o_B & -2640.28194134509 & 0.4603861 & -2640.5187532962 \\
Fe2+5_6h2o_Th & -1722.29892191805 & 0.1533699 & -1722.60062506233 \\
Fe3+6_18h2o_B & -2639.84583695427 & 0.4518076 & -2640.33354858166 \\
Fe3+6_6h2o_Th & -1721.70085490852 & 0.1492306 & -1722.37584713244 \\
Mn2+6_18h2o_B & -2527.5717524337 & 0.4593741 & -2527.80830703874 \\
Mn2+6_6h2o_S6 & -1609.59433466649 & 0.1492925 & -1609.8935103822 \\
Mn3+5_18h2o_B & -2527.12685374226 & 0.4538615 & -2527.61523417017 \\
Mn3+5_6h2o_Ci & -1608.9709480721 & 0.1487114 & -1609.65060616763 \\
\hline & & & \\
\hline
\end{tabular}


Table S2 (continued)

\begin{tabular}{llll}
\hline File name & DFT energy & ZPE & DFT + COSMO \\
\hline Ni2+3_18h2o_B & -2884.9276736991 & 0.4594282 & -2885.16497860186 \\
Ni2+3_6h2o_Ci & -1966.94121003378 & 0.1490518 & -1967.2486833776 \\
Ni3+2_18h2o_B & -2884.44396041621 & 0.45123 & -2884.9347869568 \\
Ni3+2_6h2o_Ci & -1966.27371060827 & 0.1508481 & -1966.96814571138 \\
Ni3+4_18h2o_B & -2884.43163642505 & 0.4489186 & -2884.92153090864 \\
Ni3+4_6h2o_Ci & -1966.27155027146 & 0.1463166 & -1966.94929439065 \\
Sc2+2_18h2o_B & -2137.22707904021 & 0.4524503 & -2137.46260886423 \\
Sc2+2_6h2o_S6 & -1219.25171047355 & 0.149348 & -1219.54924138581 \\
Sc3+1_18h2o_B & -2136.89787028186 & 0.4580003 & -2137.38232241375 \\
Sc3+1_6h2o_S6 & -1218.76588934351 & 0.1481795 & -1219.43054256129 \\
Ti2+3_18h2o_A & -2226.21751491347 & 0.4576389 & \\
Ti2+3_18h2o_B & -2225.98130109708 & 0.4595017 & -2226.21751491347 \\
Ti2+3_6h2o_Ci & -1307.99611427094 & 0.1564788 & -1308.29575366238 \\
Ti3+2_18h2o_A & -2225.61108435018 & 0.4614265 & \\
Ti3+2_18h2o_B & -2225.61336589405 & 0.4629661 & -2226.10027082681 \\
Ti3+2_6h2o_C3 & -1307.47027762032 & 0.1517658 & -1308.14157633931 \\
V2+4_18h2o_A & -2320.51091309617 & 0.4576923 & \\
V2+4_18h2o_B & -2225.98130109708 & 0.4614265 & -2226.21751491347 \\
V2+4_6h2o_Th & -1307.99611427094 & 0.1564788 & -1308.29575366238 \\
V3+3_18h2o_A & -2320.13443774941 & 0.4537294 & \\
V3+3_18h2o_B & -2225.61336589405 & 0.4629661 & -2226.10027082681 \\
V3+3_6h2o_Ci & -1307.47027762032 & 0.1517658 & -1308.14157633931 \\
\hline
\end{tabular}

